\title{
Féeries
}

Études sur le conte merveilleux, XVII ${ }^{\mathrm{e}} \mathrm{XIX} \mathrm{X}^{\mathrm{e}}$ siècle

Le conte, les savoirs

\section{La réfraction des sciences dans le conte de fées}

Sciences Refracted in the Fairy Tale

\section{Anne Defrance}

\section{(2) OpenEdition}

\section{Journals}

Édition électronique

URL : http://journals.openedition.org/feeries/701

DOI : $10.4000 /$ feeries.701

ISSN : 1957-7753

\section{Éditeur}

UGA Éditions/Université Grenoble Alpes

\section{Édition imprimée}

Date de publication : 1 juillet 2009

Pagination : 63-86

ISBN : 978-2-84310-140-3

ISSN : $1766-2842$

\section{Référence électronique}

Anne Defrance, "La réfraction des sciences dans le conte de fées », Féeries [En ligne], 6| 2009, mis en ligne le 15 septembre 2010, consulté le 07 septembre 2020. URL : http://journals.openedition.org/ feeries/701; DOI : https://doi.org/10.4000/feeries.701

\section{(c) Féeries}




\title{
LA RÉFRACTION DES SCIENCES DANS LE CONTE DE FÉES
}

\begin{abstract}
1
quelque point que les contes ornent l'esprit, et quelque agréables, ou quelque sublimes que soient les connaissances et les idées qu'on y puise, il est dangereux de ne lire que des livres de cette espèce. Il n'y a que les personnes vraiment éclairées, au-dessus des préjugés, et qui connaissent le vide des sciences, qui sachent combien ces sortes d'ouvrages sont utiles à la société, et combien l'on doit d'estime et même de vénération aux gens qui ont assez de génie pour en faire, et assez de force dans l'esprit pour s'y dévouer, malgré l'idée de frivolité que l'orgueil et l'ignorance ont attachée à ce genre. Les importantes leçons que les contes renferment, les grands traits d'imagination qu'on y rencontre si fréquemment, et les idées riantes dont ils sont toujours remplis, ne prennent point sur le vulgaire, de qui l'on ne peut acquérir l'estime qu'en lui donnant des choses qu'il n'entende jamais ${ }^{\mathrm{I}}$.
\end{abstract}

Ainsi l'incipit iconoclaste du Sopha (I742) oppose-t-il diamétralement conte et science, son narrateur renversant ironiquement préjugés et lieux communs pour réhabiliter le premier au déficit de la seconde, redorer l'image du conte pour en faire le flambeau des Lumières, le lieu du plaisant, du plein, du fort, du grand et de l'utile, la science étant, quant à elle, reléguée dans le territoire obscur du vide, du plat, de l'ennuyeux, parfois du dangereux : vanité par excellence, seule prisée des vaniteux. Ce propos résonne comme un lointain écho des attaques portées depuis la fin du $\mathrm{XVII}^{\mathrm{e}}$ siècle contre ces contes de vieilles que d'aucuns prétendaient à l'usage réservé des enfants et des femmes, et qui avaient l'audacieuse prétention d'accéder à un statut plus noble, sous la plume des Perrault, d'Aulnoy, de Murat, et de quelques autres. Propos qui témoigne surtout, quarante ans plus tard, de cette fracture entre deux univers toujours sentis comme antagonistes : le conte s'assumant comme fable, fiction ludique, discours mensonger, subjectif, sauvé par une intention moralisante, la science étant quête de vérités objectives, intemporelles.

I. Claude Crébillon, Le Sopha, conte moral, dans Euvres complètes, tome 2, éd. J. Sgard, Paris, Classiques Garnier, 2000, p. 282.

Féeries, n 6, 2009, p. 63-86. 
Mais la frontière est-elle totalement imperméable entre conte de fées et science ? Le renversement plaisant opéré par le narrateur du Sopha pourrait induire, paradoxalement, qu' un système de vases communicants existe entre eux. À l'époque des Lumières, les limites ne sont pas encore clairement tracées entre sciences et belles-lettres, sciences occultes et sciences "officielles », pas plus qu'entre savants professionnels et amateurs; les questions scientifiques passionnent le public et les cercles de sociabilité font se côtoyer des célébrités dans ces divers domaines, aussi bien que des amateurs éclairés. Par ailleurs, les philosophes (Rousseau, Diderot, par exemple) ne dédaignent pas d'écrire, occasionnellement, des contes de fées. Aussi ne s'étonnera donc pas que les contes reflètent ou plutôt réfractent - car ils le font à leur manière, distancée, parfois ostensiblement ludique, voire franchement critique - les sujets qui font palpiter les esprits et défrayent la chronique. Loin de prétendre épuiser le sujet des rapports entre contes de fées et sciences, nous nous bornerons ici à développer quelques exemples qui nous paraissent représentatifs de leurs relations.

\section{Voir, savoir, pouvoir : vouloir comprendre et expliquer le monde}

Dans les contrées merveilleuses, les puissances que sont les fées, les magiciens et les génies ont toujours incarné ce fantasme d'un savoir suprême, d'un pouvoir sur le monde, qui n'a pas attendu le siècle des Lumières pour se manifester, prenant au fil des temps et des cultures, de multiples formes. La fée, depuis le Moyen Âge, est vue comme un " abrégé de science profonde ${ }^{2}$ ", même si cette science est aussi, souvent, limitée, mise en échec par l'existence de forces antagonistes, qu'elles émanent de puissances rivales de nature surnaturelle, ou tout simplement de l'amour humain, dont les premiers contes littéraires se plaisent à exalter la suprématie. Dans de nombreux contes, ce sont les fées qui guérissent la stérilité frappant les couples royaux, compensant ainsi l'insuffisance de la science, des médecins et chimistes de profession ${ }^{3}$. Comme leurs ancêtres les Parques qui tissent le fil de la destinée humaine, elles se tiennent à l'origine de la vie, occupent une position divine. Fées et magiciens savent donc aussi déchiffrer le Grand livre, ou livre des Destins, tirer les horoscopes, à une époque où

2. "À Madame la première présidente de Metz. Épître gauloise sur un conte des fées ", dans [Anon.], Nouveau conte des fées. Le portrait qui parle (I699), Paris, Champion, "B.G.F. ", vol. 4, 2005, p. 772.

3. Par exemple, dans Blanche, [Anon.], Recueil de contes galants, I699. 
l'on confond encore souvent astrologie et astronomie ${ }^{4}$. Elles défient les lois du temps et de l'espace, et leurs merveilleuses bottes de sept lieues, chariots et autres engins volants, agents de leur quasi-ubiquité (autre qualité divine) préfigurent les inventions techniques des temps futurs, bien avant celle de la Science-fiction.

Le grand élan vers le savoir qui soulève le siècle des Lumières, transparaît dans les contes. Pour exemple, cet éloge émanant d'un roi amateur de Physique chez Crébillon :

Ah! Monsieur ! que c'est surtout, une belle chose, que cette Physique ! Que les objets qu'elle se propose sont grands! Qu'elle élève l'esprit ! En effet ! quoi de plus digne de l'homme que d'étudier la structure de l'univers, les causes de tout ce qu'il offre à nos yeux, de percer les entrailles de la terre, d'y arracher à la nature les secrets qu'elle a cachés, et de voler de là au plus haut des cieux, y observer la marche de ces corps immenses et innombrables, que leur éloignement n’a pu dérober à notre curiosités!

Cet enthousiasme scientifique n'est guère éloigné du rêve cabalistique, qui imprègne aussi le conte, et dont témoigne cette féerique proposition, chez $\mathrm{M}^{\text {me }} \mathrm{d}^{\prime}$ Aulnoy :

À quoi me servirait d'être lutin ? - À mille choses utiles et agréables, repartit la fée ; vous êtes invisible quand il vous plait ; vous traversez en un instant le vaste espace de l'univers; vous vous élevez sans avoir des ailes : vous allez au fond de la terre sans être mort, vous pénétrez les abîmes de la mer sans vous noyer ; vous entrez partout, quoique les fenêtres et les portes soient fermées $[\ldots]^{6}$.

Certains objets magiques ou châteaux féeriques réalisent, dès les premiers contes de la fin du XVII ${ }^{\mathrm{e}}$ siècle, ce rêve encyclopédique qui hantera les Lumières. Ainsi, dans La Chatte blanche de $\mathrm{M}^{\mathrm{me}} \mathrm{d}$ 'Aulnoy, est décrite " une pièce de toile de quatre cents aunes si merveilleuse, que tous les oiseaux, les animaux et les poissons y étaient peints avec les arbres, les fruits et les plantes de la terre, les rochers, les raretés et les coquillages de la mer, le soleil, la lune, les étoiles, les astres et les planètes des cieux ${ }^{7}$ ". De même, sur des dentelles ouvrées de main de fée, dans La Biche au bois, toute l'histoire du monde est représentée. Quand quelques années plus tard, les Mille et Une Nuits de Galland ouvrent leurs horizons lointains aux lecteurs du siècle naissant, de nouveaux savoirs s'y engouffrent : nombre

4. Ainsi $\mathrm{M}^{\mathrm{me}}$ de Villeneuve, à plusieurs reprises, dans ses contes de $\mathrm{1} 740-1745$.

5. Claude Crébillon, Ah quel conte! Conte politique et astronomique, éd. R. Jomand-Baudry, dans Euvres complètes, éd. citée, tome 3, p. 428-429.

6. $\mathrm{M}^{\mathrm{me}}$ d'Aulnoy, Contes des fées, éd. N. Jasmin, Paris, Champion, « B.G.F. ", vol. I, 2004, p. 228.

7. Ibid., p. 770. 
de recueils de contes orientaux ou orientalisants accueillent des notes de bas de page parfois copieuses, nourries d'informations puisées, entre autres, dans la Bibliothèque orientale d'Herbelot, offrant au lecteur curieux une information lexicale, géographique, ethnographique, religieuse, zoologique, etc. Le vaste monde, dont les limites ne cessent d'être repoussées et les secrets dévoilés par les voyages d'exploration et les missions jésuites, suscite la curiosité, le goût pour l'Orient. Ces notes infrapaginales, quasiment absentes du recueil de Galland (sauf des Voyages de Sindbad qui ont constitué un recueil antérieur autonome avant que les récits regroupés sous ce titre ne soient rattachés à l'ouvrage), sont plus nombreuses encore chez ses successeurs, Pétis, Bignon, Gueullette.

L'abbé Bignon constitue un cas particulièrement intéressant : directeur de toutes les Académies, il présida pendant quarante ans celle des Sciences, et fut, de ce fait, à l'origine de nombreuses missions scientifiques menées à l'étranger, protégeant par exemple à distance celles de Tournefort et de Réaumur ${ }^{8}$. Il fut aussi membre du comité de direction du Journal des Savants pendant de longues années. On imagine donc les relations qu'il devait nécessairement cultiver avec eux, ainsi qu'avec les voyageurs et missionnaires, sans fréquenter pour autant, dit-on, les salons mondains. Son unique œuvre littéraire, les Aventures d'Abdalla, publiée entre I7I2 et I7I4, réalise «l'étonnante interpénétration des références les plus concrètes et des fictions les plus merveilleuses ${ }^{9}$ ". Son auteur y fait voler en éclats la représentation fallacieuse que nous nous faisons, nous, lecteurs des temps modernes, de ces catégories prétendument antagonistes que sont le rationnel et le merveilleux, le plaisant et le sérieux. Dans ces récits fictifs très documentés, Bignon dispense une information historique et géographique extraordinairement précise, là où Galland restait très évasif, note R. Robert. Ainsi, des explications sur le comportement des cachalots, sur les méthodes de récolte de leurs déjections par les indigènes ${ }^{10}$, sont fournies dans ces récits mus par une évidente intention didactique. Le conte merveilleux oriental représente plus qu'une fenêtre ouverte sur le monde : c'est une sorte de longue-vue, de lunette - ainsi l'appelait-on - que le lecteur, à l'instar des explorateurs abordant des terres nouvelles, est invité à déplacer horizontalement, au rythme du grand périple du héros traversant l'Inde, l'actuelle Indonésie et la Perse.

8. Voir la notice de l'édition de R. Robert des Aventures d'Abdalla de J.-P. Bignon, Paris, Champion, «B.G.F. », vol. 8, 2006.

9. Ibid., p. 918.

Io. Ibid., p. 912-9I4. 
La lunette terrestre, inventée en I609 par Galilée, avait connu en un siècle une considérable évolution. Les instruments d'optique faisaient d'ailleurs tous l'objet d'une véritable fascination, perceptible dans plusieurs contes de fées. Chez Pajon, dans un long conte publié en I740, l'Histoire du Prince Soly surnommé Prenany et de la Princesse Fêlée, une reine prend pour cinquième mari

un homme de mérite [...] avait rendu de grands services à l'État par l'invention des lunettes qu'il avait trouvée (il avait fait cette admirable découverte en regardant au travers d'une bouteille). Il en avait fait qui approchaient la Lune, et la faisaient paraitre plus grande, ce qui lui avait attiré l'affection du peuple. Il en faisait d'autres qui grossissaient les objets, et qui servaient à la reine, dont la vue s'était fort affaiblie à force de pleurer les mépris de ses premiers époux ${ }^{\mathrm{II}}$.

L'instrument de vue a beau dater, en fait, du Moyen Âge, il est semble-t-il, dans ce conte qui le réinvente, un objet suffisamment précieux pour que la reine préfère laisser tomber vraiment le nouveau-né qu'elle porte dans les bras, pour avoir les mains libres de retenir sa paire de lunettes qui menace seulement de glisser.

On a évoqué plus haut certains objets magiques aptes à satisfaire une pulsion de savoir total, objets nés de la plume de la pionnière qu'est $\mathrm{M}^{\mathrm{me}} \mathrm{d}$ 'Aulnoy. Ils ont vraisemblablement inspiré Marguerite de Lubert, qui publie en 1743 La Princesse Lionnette et le Prince Coquerico. Ce conte narre les aventures d'un jeune homme élevé par la fée Cornue, dans un palais " bâti d'une seule opale, si transparente et si belle qu'à travers des murs on voyait un grain de mil au bout du jardin ${ }^{12} »$. Cet espace architectural qu'est le château merveilleux constitue donc ici un véritable dispositif optique, ses murs faisant office de lentilles transformant leurs habitants en grands voyants. Aussi ce lieu est-il ici la métonymie du pouvoir féerique, qui ne se limite plus au pouvoir métamorphique (même si les fées de $\mathrm{M}^{\text {lle }}$ de Lubert ne dédaignent pas de faire usage, loin s'en faut, de ce tour de passe-passe conventionnel, en débordant d'imagination). Si la métamorphose masque l'identité des êtres, les confisque à eux-mêmes et au regard d'autrui ${ }^{13}$, au contraire, ce château de cristal a pour fonction de révéler,

II. H. Pajon, Histoire du Prince Soly surnommé Prenany et de la Princesse fêlée, dans Hamilton et autres conteurs, éd. A. Defrance et J.-F. Perrin, Paris, Champion, "B.G.F. ", vol. I7, 2008, p. 82I. (Les contes de Pajon, Duclos, Cazotte cités dans cet article réferent à ce volume et sont édités par mes soins.)

I2. Marguerite de Lubert, Contes, éd. A. Zygel-Basso, Paris, Champion, « B.G.F. », vol. I4, 2005, p. 249.

I3. On pense à Tecserion : l'identité du héros Mélidor est presque jusqu’à la fin cachée au lecteur, et révélée au terme d'une série de métamorphoses, du monstre à l'homme. 
de dévoiler le monde en repoussant les limites naturelles de l'œil humain. Cet espace matérialise ce don de vue surnaturel que possèdent les fées, et qu'elles veulent parfois offrir à leurs protégés. Si les premiers châteaux de fées regorgeaient de pierres précieuses, d'objets d'art et de richesses incommensurables, aptes à satisfaire une soif de possession matérielle, un fantasme de luxe inouï, celui-ci laisse entendre que le désir du lecteur s'est déplacé. Ce n'est plus alors la nostalgie d'un âge d'or et des fastes aristocratiques louis-quatorziens qui sont exaltés par ce conte, mais le savoir, l'appétit de connaissances. Les méthodes préconisées pour y accéder sont celles que valorisent les savants à l'époque : l'observation et l'expérience. De lieu merveilleux banal qu'il était, le palais féerique a donc ici totalement changé de statut : il est devenu objet scientifique, observatoire, œil immense ; il magnifie la connaissance en englobant intégralement le sujet de l'observation, le prince enfant qui y est enfermé. Coquerico y apprend le monde avant de s'y engouffrer. Il y prépare, tout en s'amusant, son futur métier de roi, passant quotidiennement trois heures dans une "galerie magnifique, remplie de cartes, de mappemondes, d'instruments de géométrie, de plans en relief des plus belles villes de l'Asie, de l'Europe et de l'Afrique ${ }^{\mathrm{I4}}$ ». Galerie qui possède de surcroît " un plan en relief de l'univers entier ", suscitant l'admiration du narrateur :

Quel art était dans ce fameux ouvrage! Non seulement les royaumes et leurs provinces, jusques aux moindres habitations, mais tous les hommes qui couvrent la terre y remplissaient chacun leur emploi ; ils parlaient chacun leur langue, on les entendait, on les voyait ; les choses les plus secrètes y étaient dévoilées, la mer et ses vaisseaux, les rivières, les lacs, les plus petits ruisseaux, les déserts jusques aux terres inconnues : rien n'étant caché à la savante Cornue, elle les avait décrites dans son plan ${ }^{15}$.

Ce conte reflète l'intérêt pour la cartographie, qui se développe tout au long du siècle, et motive de nombreuses expéditions dans le monde entier, la correction de la carte du monde étant une tâche que se sont fixée l'Académie des Sciences et l'Observatoire. J.-D. Cassini, son directeur, a fait dresser, en 1696, un très grand planisphère sur lequel sont pour la première fois correctement situées les masses des continents. Quant aux cartes merveilleuses imaginées par $\mathrm{M}^{\mathrm{lle}}$ de Lubert, elles forment une véritable encyclopédie vivante en trois dimensions. Le personnage peut y contempler tout à son aise "des palais où les hommes et les femmes de chaque nation [...] étaient habillés selon leurs coutumes; et par l'art de

I4. Ibid., p. 250.

15. Ibid., p. 25 I. 
la fée, ils allaient et venaient et parlaient chacun leurs langues et discouraient selon leur état ${ }^{16}$ ", sous la forme de figures "pas plus haute[s] que le doigt " que le narrateur compare à de " jolies marionnettes ${ }^{17}$ " (ce qui laisse supposer qu'il peut aussi les toucher, ce que le conte ne dit pas). L'artifice merveilleux de la miniaturisation qui traduit le goût de l'époque pour les automates, poupées, marionnettes, objectivisant les individus observés, sert un fantasme de possession et de toute puissance ${ }^{18}$. Au modèle de la lunette s'ajoute en filigrane, ici, celui du microscope. Au fils des séances studieuses passées dans la galerie de l'univers, le jeune prince développe sa réflexion et son jugement; il comprend l'art de la guerre, sur terre et sur mer, et assiste même à l'opéra et à la comédie. "Comme il avait du goût pour les choses d'esprit, il allait à toutes les harangues de l'Académie et en jugeait fort bien ${ }^{19}$."

Un tel dispositif (à la fois optique et acoustique) pourrait facilement nourrir quelques fantasmes voyeuristes, mais l'auteur a décidé de n'asservir ce matériel cartographique à d'autres fins que pédagogiques. L'idée affleure néanmoins : quand plus tard le jeune prince, qui est sorti du palais, est tombé amoureux et recherche sa bien-aimée sur ses plans, "il n'osa regarder dans le moment qu'elle s'allait mettre au lit [...] il n'aurait même rien vu, car elle éteignit la lumière et l'obscurité lui aurait tout dérobé $^{20}$ ». Faut-il rappeler que pulsion scopique et pulsion de savoir sont des pulsions sexuelles, qui dévient de leur objet, et sont inhibées quant au but, pour reprendre la terminologie freudienne ? Ce but peut toujours refaire surface, le conte le montre à sa manière, l'hypothèse niée dans la phrase citée laissant percevoir la trace du refoulement.

Dans un conte paru trois ans plus tôt, La Belle et la Bête, l'héroïne de $\mathrm{M}^{\mathrm{me}}$ de Villeneuve, enfermée elle aussi dans un palais enchanté et installée dans une pièce sombre, peut assister à un spectacle de théâtre grâce à un astucieux dispositif, qui ne requiert pas cette fois la miniaturisation des objets et des individus. Il ne s'agit plus ici pour l'héroïne d'apprendre, mais de tromper son ennui dans un palais où elle-même est vue et surveillée, sans qu'elle le sache, par des yeux désirants - ceux de la bête - et par une fée, grande ordonnatrice, qui supervise l'ensemble :

16. Idem.

17. Ibid., p. 252-253.

I8. D'autres contes de $\mathrm{M}^{\text {He }}$ de Lubert, La Princesse Camion, par exemple, agitent pudiquement, ce fantasme, qui libère évidemment tout son potentiel érotique dans le conte libertin (La Poupée de Bibiena, par exemple).

I9. Ibid., p. 253.

20. Ibid., p. 257. 
Cette pièce était percée de quatre fenêtres de chaque côté [... La Belle] ne trouva qu'une ouverture, qui donnait sur un endroit fermé. Ce lieu, quoique spacieux lui parut obscur, et ses yeux ne purent apercevoir qu'une lueur éloignée, qui ne semblait venir à elle qu'au travers d'un crêpe extrêmement épais. [...] Une vive clarté vint tout à coup l'éblouir. On leva la toile, et la Belle découvrit un théâtre des mieux illuminé [...]. Curieuse de voir de quelle étoffe était le tapis de la loge voisine de la sienne, elle en fut empêchée par une glace qui les séparait, ce qui lui fit connaître que ce qu'elle avait cru réel n'était qu'un artifice, qui par le moyen de ce cristal réfléchissait les objets et les lui renvoyait de dessus le théâtre de la plus belle ville du monde. C'est le chefd'œuvre de l'optique de faire réverbérer de si loin ${ }^{21}$.

Ce clin d'œil final pointe malicieusement la transgression des limites de la science humaine, pour exalter le merveilleux. Le dispositif décrit combine poétiquement deux, voire trois appareils optiques. Le premier, le plus évident, est celui de la camera obscura ${ }^{22}$, fort ancien, déjà évoqué par Aristote, et qui est à l'origine de l'appareil-photo des temps futurs. Le second serait le périscope, qui permet de voir sans être vu, mais alors il faudrait en supposer un qui soit placé au-dessus de la Comédie française, vraisemblablement désignée ici, et un autre au-dessus de la pièce où se trouve la Belle (éventuellement aussi un troisième dont jouirait la Bête qui l'observe en même temps). Troisième et dernier appareil : la lunette, encore une fois. Celle de Galilée, "lunette terrestre », permettait d'obtenir une image droite et non inversée, alors que la lunette astronomique, ou télescope, inventé en I668 par Newton, nécessitait, pour redresser l'image, l'intégration d'un deuxième miroir incliné à $45^{\circ}$. Le narrateur n'entre pas dans de tels détails techniques dans cette composition syncrétique, mais avec une précision descriptive encore inégalée dans le genre, il nomme les matériaux utilisés (" un crêpe extrêmement épais ", " une glace », " ce cristal ") ainsi qu’il définit le procédé optique (" réfléchissait les objets », " renvoyait de dessus ", détail qui implique l'inclinaison du miroir. En inventant le système de retransmission en temps réel d'un spectacle éloigné dans l'espace, d'un espace clos à un autre, $\mathrm{M}^{\mathrm{me}}$ de Villeneuve anticipe ici sans le savoir sur une autre invention qui verra le jour deux siècles plus tard, celle de la télévision, permettant le reportage en direct.

21. $\mathrm{M}^{\mathrm{me}}$ de Villeneuve, La Jeune Américaine et les contes marins, éd. É. Biancardi, Paris, Champion, «B.G.F. », vol. I5, 2008, p. I29.

22. Sur ce dispositif, voir A. Gaillard, "Anamorphoses : les lieux d'illusion picturale dans la fiction (contes et romans) au XviII ${ }^{e}$ siècle", dans Locus in fabula, La topique de l'espace dans les fictions françaises d'Ancien Régime, études réunies et présentées par Nathalie Ferrand, Louvain-Paris, Éd. Peeters, 2004, p. 539-552. 
Dans la dernière phrase du texte cité précédemment, on pourrait également entendre comme l'écho d'une remarque admirative proférée par un amateur en visite dans l'un de ces nombreux cabinets de curiosités fréquentés par les hommes et les femmes du XviII ${ }^{\mathrm{e}}$ siècle, et dans lesquels étaient exposés des instruments optiques, qui alors valaient un grand prix (loupes, microscopes simples, miroirs et prismes, lentilles, et peu à peu, microscopes composés). L'auteur, toujours prompt à magnifier l'étude, aime évoquer ces espaces de prédilection que sont ces cabinets et les bibliothèques. Ainsi Mirliton, conte encastré dans le récit Les Belles solitaires ${ }^{23}$, narre les aventures d'une jeune reine enfermée dans un château désert, garni de «trésors et de raretés [... et de] toutes les curiosités qui se peuvent exprimer, qui remplissaient plusieurs galeries et plusieurs cabinets ${ }^{24} »$ :

Enfin, généralement, tous les agréments de la vie solitaire se rencontraient en cette demeure, et le génie, car c'en était un qui avait bâti ce château, avait eu la précaution de n'y excepter rien des commodités de la vie. Il y avait mis une bibliothèque, des instruments de mathématique, des livres de musique, des armes pour la chasse, les choses nécessaires à la pêche, enfin jusqu'aux outils de toutes sortes de professions, afin que rien ne manquât au soulagement des infortunés qui y étaient conduits ; et on n'aurait pu se trouver malheureux dans ce lieu solitaire, sans la perte de la liberté, qui seule fait le vrai bien. Mais il fallait absolument s'y suffire à soi même, n'ayant personne à qui parler, ni aucune communication dehors ${ }^{25}$.

L'héroïne fait bon usage de cette prison et dans sa retraite studieuse (comparable à certains égards à celle vécue par l'auteur), elle compose " plusieurs beaux traités, sous le titre De la connaissance et de l'Usage de la vraie libertée ${ }^{26}$ ". Qu'une femme, ici une simple mortelle, incarne cette figure du sage anachorète, et écrive des traités de philosophie au milieu de livres et d'instruments de mathématique, n'est pas coutume dans le genre féerique. On n'en rencontrait pas dans les contes de fées de la fin du XVII ${ }^{\mathrm{e}}$ siècle, même si depuis longtemps déjà les femmes tenaient salon. À la Cour, celui de la duchesse du Maine (appelé « la cour de Sceaux) réunit, dans sa deuxième vie, après 1720 , aussi bien savants qu'hommes de lettres autour de Fontenelle. Voltaire y composa quelques contes (Zadig et Micromégas). À Paris, celui de la marquise de Lambert, ouvert de I7ı à sa mort en I733, était, disait-on, l'antichambre de l'Académie française.

23. É. Biancardi pense que les contes insérés dans ce récit-cadre ont été écrits durant les années 1720.

24. $\mathrm{M}^{\mathrm{me}}$ de Villeneuve, Les Belles solitaires, éd. É. Biancardi, Champion, "B.G.F.", vol. I5, p. 827 .

25. Ibid., p. 823 .

26. Ibid., p. 824 . 
Il accueillait gens du monde, gens de lettres et savants. On pourrait encore citer celui de $\mathrm{M}^{\mathrm{me}}$ de Tencin qui, à partir de $\mathrm{I726}$, reçoit Montesquieu, Marivaux, et des conteurs comme Duclos et Marmontel $\left(\mathrm{M}^{\mathrm{me}}\right.$ Geoffrin prendra la relève en I750, s'entourant d'artistes et des philosophes encyclopédistes). Peu avant que $\mathrm{M}^{\mathrm{me}}$ de Villeneuve ne publiât ce texte (I745), Émilie du Châtelet, qui présidait une société fréquentée par des savants et des hommes de lettres faisait paraître ses Institutions de physique, et son Analyse de la Philosophie de Leibnitz (1740). Les sources d'inspiration ne manquaient donc pas.

Aussi, nombreux sont les contes de fées des années 1740 qui évoquent ces cercles savants (surtout pour les railler, on le verra un peu plus loin) et témoignent de l'engouement des femmes comme des hommes pour les sciences et de leur diffusion, ainsi que pour les progrès techniques qui en résultent. Dans un conte des Mille et Un Jours (I7IO-I715), l'Histoire de Malek et de la Princesse Schirine, on croise un véhicule volant (il s'en trouvait déjà dans les utopies, dans l'Histoire comique des États et empires de la lune de Cyrano de Bergerac par exemple). Cet objet merveilleux est de conception purement artisanale. L'auteur retrace le travail de l'ouvrier, plaçant les ressorts et perfectionnant l'objet, puis les essais du véhicule : "On le couvrit d'un tapis de Perse, [...] l'étranger [...] entra dedans. En même temps, le coffre s'éleva de terre et fendit les airs avec une vitesse incroyable ${ }^{27}$. " L'homme offre ensuite le véhicule au héros, et ce scénario confirme bien le statut mitoyen, transitoire, de cet objet merveilleux, à la lisière du normal et du surnaturel : le tapis qui évoque le tapis volant des coffres orientaux (ce conte en est un), puis le don de l'objet (topos féerique) achève de le charger de connotations. Le donateur explique alors au personnage :

Ne vous imaginez pas [...] qu'il y ait de l'enchantement dans de ce que vous venez de voir. Ce n'est point par des paroles cabalistiques ni par la vertu d'un talisman que ce coffre s'élève en l'air. Son mouvement est produit par l'art qui enseigne les forces mouvantes. Je suis consommé dans les mécaniques, et je sais faire encore d'autres machines aussi surprenantes que celle- $\mathrm{ci}^{28}$.

Cette précision est censée désenchanter l'objet, en magnifiant la technique et le savoir (dont la conjugaison est appelée " art "). Il ne reste ensuite, qu'à délivrer le mode d'emploi de la machine : "En tournant cette vis, vous irez à droite et en tournant celle-là, vous irez à gauche ; en

27. Pétis de la Croix, Les Mille et Un Jours, éd. C. Bahier-Porte, Paris, Champion, «B.G.F. », vol. 8,2006, p. 633 .

28. Ibid., p. 636-634. 
touchant ce ressort, vous descendrez ${ }^{29}$. " Ce conte, ainsi que celui de $\mathrm{M}^{\text {me }}$ de Villeneuve, sont bien représentatifs d'une certaine « rationalisation du merveilleux $^{30}$ " qui affecte le genre, et qui cohabite toujours avec un merveilleux de type traditionnel.

Les lointains ancêtres des héros de contes, les dieux et déesses de la mythologie, ne disposaient pas de tels véhicules pour se déplacer, ni de l'«art " d'en apprendre la conduite aux humains.

\section{La quête des origines et des causes}

À l'orée du siècle des Lumières, les astres, qui portaient le nom de ces dieux, se partageaient avec eux l'intérêt d'un philosophe, Fontenelle, auteur à la fois des Entretiens sur la pluralité des mondes (I686), et De l'Origine des fables, traité publié en I724, mais vraisemblablement connu bien plus tôt.

Réfléchissant aux raisons qui ont amené les hommes à se repaître de fictions, Fontenelle fait ressortir, dans ce traité, le lien existant entre science et Fable :

Toutes les métamorphoses sont la physique des premiers temps. Les mûres sont rouges, parce qu'elles sont teintes du sang d'un amant et d'une Amante ; la perdrix vole toujours terre à terre, parce que Dédale, qui fut changé en perdrix, se souvenoit du malheur de son fils qui avoit volé trop haut ; et ainsi du reste ${ }^{31}$.

Aux mystères de la nature, l'imaginaire humain s'est toujours attelé à trouver un sens. Aussi, l'admiration des phénomènes physiques qui frappaient déjà l'homme primitif, l'amenant à élaborer dans la nuit des temps une explication poétique du monde, l'apparente au savant moderne, travaillé par des inquiétudes, des questionnements comparables. Le philosophe qui prend de la distance pour analyser les tenants et aboutissants de cette humaine curiosité, cette libido sciendi (Fontenelle en avait exalté les attraits et les vertiges en termes imagés et poétiques dans les leçons d'astronomie de ses Entretiens), œuvre à son tour, ici, à la construction des savoirs, et le revendique :

29. Ibid., p. 634 .

30. J'emprunte le mot à Christelle Bahier-Porte, ibid., p. 256.

31. B. le Bovier de Fontenelle, De l'origine des fables, [en ligne] disponible sur <http://www. eliohs.unifi.it/testi/70o/fontenelle/fables.htm> (consulté le Is mai 2009), Html edition for Eliohs by Guido Abbattista (mars 1998), non paginé. 
Ce n'est pas une science de s'être rempli la tête de toutes ces extravagances des Phéniciens et des Grecs ; mais c'en est une de savoir ce qui a conduit les Phéniciens et les Grecs à ces extravagances ${ }^{32}$.

Aussi les auteurs de contes de fées, dans la lignée des anciens mythes et des vieilles légendes, continuent-ils de frayer avec cette pulsion de savoir quand ils se plaisent, pareillement, à expliquer l'origine des choses, à dévoiler à leurs lecteurs l'explication d'un phénomène. Mais ils le font à partir d'un autre point de vue, puisqu'ils adoptent cette posture postcritique qui consiste à introduire dans leur propos une distance humoristique absente des essais savants.

On ne finirait pas de recenser les exemples de cette frénésie qui s'empare du genre, dès les premiers conteurs et dévoile l'origine de tout et de n'importe quoi. Elle ne cesse de s'exprimer tout le siècle et se manifeste dans des séquences plus ou moins étendues de récits, de la simple allusion ponctuelle en passant par une séquence narrative conséquente, pour recouvrir, parfois même, le conte tout entier : $\mathrm{M}^{\mathrm{me}} \mathrm{d}$ 'Auneuil publie L'inconstance punie ou L'origine des cornes (1702), L'origine de l'occasion (1703). Le Prince Périnet ou L'origine des pagodes est publié anonymement en I73I. Ces contes pastichent le discours mythique tout en parodiant les dissertations savantes : fictions et écriture didactique se rejoignant alors - superficiellement - en une même démarche étiologique, qui prend un tour particulièrement satirique dans la féerie post hamiltonienne, et surtout dans les années I734-I760, avec Claude Crébillon et ses épigones.

Je ne citerai ici qu'un exemple, humoristique, de ce topos de l'origine $^{33}$, il concerne une fois encore l'optique. On a vu Pajon réinventer les lunettes. Charles Duclos en propose une autre explication, plus fantaisiste encore, dans Acajou et Zirphile. Il en renverse totalement l'usage ordinaire :

La vertu des lunettes était, en affaiblissant la vue, de tempérer la vivacité de l'esprit par la relation de l'âme et du corps. Voilà la première invention des lunettes; on les a depuis employées pour un usage tout opposé, et c'est ainsi qu'on abuse de tout. Ce qui prouve cependant combien les lunettes nuisent à l'esprit, c'est de voir que de vieux surveillants sont tous les jours trompés par de jeunes amants sans expérience, et l'on ne peut s'en prendre qu'aux lunettes ${ }^{34}$.

32. Idem.

33. Je développe ces points dans un autre article : «Fictions de l'origine, dans les contes de fées du classicisme aux Lumières ", dans Fictions de l'origine, colloque organisé par Christophe Martin, 4-6 juin 2009, Université Paris io.

34. C. Duclos, Acajou et Zirphile, Champion, « B.G.F. », vol. I7, p. I35I. 
Le détournement des instruments d'optique pour les asservir à la critique des mœurs n'était pas nouveau ${ }^{35}$, et une brillante illustration venait d'être fournie, en I74I, par un conte d'André-François Deslandes ${ }^{36}$.

\section{La critique des sciences et leur travestissement parodique}

Si l'attrait pour les sciences est reflété par les contes de fées, il est aussi tourné en dérision dans la production conteuse des années I740, à une époque de pleine effervescence : les partisans des théories de Newton, dont les thèses se sont répandues lentement en Europe durant les trente premières années du siècle, s'opposent vigoureusement au parti des Cassini, qui défend celles de Descartes. La théorie des tourbillons est en effet toujours officielle, même si elle vient d'être mise à mal par les résultats de l'expédition en Laponie de Maupertuis et Clairaut, de retour en France en 1737 .

Le jeune Cazotte fait son entrée en littérature en suivant son modèle, Crébillon : «Le royaume de Zinzim est un de ceux que les géographes ont perdu de vue, je crois même qu'il a changé de continent pour leur faire pièce ${ }^{37}$ ", clame d'entrée de jeu le malicieux narrateur de La Patte du chat, en I74I. Les pays de Nulle part sont légion dans les contes de fées, mais l'auteur se plaît à ajouter un coup de griffe bien appuyé :

Sans doute que quelque antiquaire, à force de sueurs, pourrait en découvrir les traces ; mais ces savants partagent leur attention entre les épitaphes et la mitraille, et c'est dommage qu'une aussi belle science se borne d'elle-même ${ }^{38}$.

L'attaque vise clairement les Académiciens (et précisément les membres de l'Académie des inscriptions). L'auteur, qui deviendra en vieillissant de plus en plus hostile au clan des Philosophes, s'en tient dans ses ouvrages de jeunesse à une attitude mesurée à leur encontre, raillant, plutôt que les savants, la sotte prétention des beaux-esprits avides de s'engouffrer dans ce qui est à la mode. Dans ce premier conte, il salue en effet le rare génie

35. Sur l'articulation de la morale et de l'optique, voir L'optique des moralistes, de Montaigne à Chamfort, textes recueillis et présentés par B. Roukhomovsky, Paris, Champion, 2005.

36. A.-F. Deslandes, L'optique des moeurs opposée à l'optique des couleurs, éd. B. Roukhomovsky, Féeries, n 2, 2004-2005, p. 273-282. Voir l'essai introductif de l'éditeur : "Des effets merveilleux de l'optique ", p. 259-272.

37. J. Cazotte, La Patte du chat, conte zinzimois, Champion, «B.G.F.", vol. I7, p. I005. Dans Tanzaï et Néadarné, Crébillon situe son intrigue "dans la grande Chéchianée, pays aujourd'hui perdu par l'ignorance des géographes " (éd. citée, tome I, p. 275).

38. Idem. 
de l'homme déjà âgé qui a introduit la philosophie dans le monde. Il vise probablement Fontenelle, secrétaire perpétuel de l'Académie à partir de I697, et qui pendant un demi-siècle, en rédige l'histoire, exposant les communications de ses membres, et écrivant leur éloge. Le narrateur du conte déplore que son personnage ait suscité, par désir d'imitation, toute une série de philosophies bizarres : "Cela allait si loin que deux Camayeuls en se saluant se souhaitaient réciproquement des lumières philosophiques ; car quiconque n'en avait pas passait pour imbécile ${ }^{39}$. " Rappelons qu'à l'époque, le terme de "philosophe " pouvait désigner tout autant celui qui étudie les sciences de la nature, que l'homme de lettres. L'engouement pour les sciences est raillé aussi par Duclos : la cour de la fée Ninette, dans laquelle évolue Acajou, est gagnée par le bel esprit, ce " travers à la mode» :

Pour éviter un certain pédantisme que donne souvent l'étude, on avait imaginé le secret d'être savant sans étudier. Chaque femme avait son géomètre ou son bel esprit, comme elles avaient autrefois un épagneul ${ }^{40}$.

Voltaire, dans une lettre à Cideville datée du I6 avril I735, fait état de cette tocade succédant à celle des Belles-lettres, qui « périssent à vue d'œil » tandis que « tout le monde commence à faire le géomètre et le physicien ${ }^{4 \mathrm{I}}$ ». Dans une lettre à Frédéric II datée de février I739, $\mathrm{M}^{\mathrm{me}}$ du Châtelet déplore les aberrations de la vulgarisation scientifique : "L'attraction n'est pas toujours bonne à toutes les sauces, et on en a un peu abusé ces temps-ci. »

Cela se vérifie évidemment chez les conteurs qui, toujours prompts à la satire, font un usage particulièrement fantaisiste des théories newtoniennes (attraction, gravitation). On attendait les résultats de l'expédition de La Condamine au Pérou ${ }^{42}$ pour achever de les confirmer, quand Cazotte publie ses Mille et une fadaises, en I742. Il s’y amuse à confondre gravité et gravitation : "Savez-vous ce que c'est que la gravitation ? " demande à Gracieux Brillandor, habitant de la lune tombé du ciel.

- Non, répondit Gracieux, je n'en sais pas un mot. - C'est quelque chose de fort joli, dit Brillandor, mais il faut trop de temps pour l'expliquer; qu'il vous suffise de savoir que, par le moyen de cette vertu, toutes les têtes pleines de cervelle gravitent vers la terre, et toutes celles qui n'en ont point vers la lune. Vous devez juger par là

39. Ibid., p. 1036.

40. C. Duclos, Acajou et Zirphile, éd. citée, p. I379.

4I. Cité par R. Jomand-Baudry dans Ah quel conte!, éd. citée, p. 714, note I54.

42. Elle sera de retour en 1743 . Mais les théories newtoniennes continueront d'être contestées en dépit des résultats des expériences, largement en leur faveur. 
que ma planète n'est peuplée que de têtes à l'évent ; aussi les habitants sont si légers que leurs pieds ne touchent pas à la lune ${ }^{43}$.

Crébillon a particulièrement tourné en dérision la science et l'engouement suscité par elle, dans son Conte politique et astronomique. Tel est le sous-titre donné à Ah quel conte! publié en I754. Il existe plusieurs versions de ce texte, la première connue étant le manuscrit de Madrid que Régine Jomand-Baudry date de 1739 . La loi de l'attraction y est également évoquée. Le roi des Autruches, qui a passé une soirée charmante avec sa bien aimée, curieux de savoir si son favori Taciturne a, de son côté, cédé aux avances d'une précieuse métamorphosée en grue, s'entend répondre que la soirée a été occupée

à des dissertations fort ennuyeuses sur le sentiment [...] quelques points de physique nous ont servi de récréation : elle est fort savante, cependant elle forge des systèmes sur lesquels je ne suis point du tout de son avis ; et quoi qu'elle en dise, son attraction ne m’est point bien démontrée ${ }^{44}$.

Un peu plus loin, on évoque encore les femmes savantes : "Il semble que l'amour même ne soit jamais pour elles, qu'une expérience de physique ${ }^{45}$. " Aussi, peut-on espérer séduire "la femme le plus sottement entêtée de science » dans un moment de " distraction ${ }^{46}$ ». La polysémie des termes "physique " ou " attraction ", ainsi que leurs déclinaisons lexicales laissent planer l'équivoque. À diverses reprises, les personnages du conte se complaisent à des jeux de mots tendancieux gazés sur le même thème. On les imagine assez bien monnaie courante à l'époque.

D'ailleurs, la dégradation parodique des théories newtoniennes était occasionnellement pratiquée par les savants eux-mêmes, à titre de plaisanterie. On a, par exemple, attribué au mathématicien Alexis Claude Clairaut, qui avait accompagné Maupertuis dans son expédition au pôle, une gauloiserie insérée dans un ouvrage collectif de la Société du Bout du Banc, et intitulée "Problème Physico-mathématique ». Il s'agit d'une démonstration pseudo-savante, décrivant de manière voilée le mécanisme de l'érection virile, graphiques géométriques à l'appui ${ }^{47}$. Pièce qui suscite cette réaction réjouie de Duclos, rédacteur de la Critique de l'ouvrage qui le clôt : «Ah ! voilà donc enfin la Géométrie appliquée à quelque chose

43. J. Cazotte, Les Mille et une fadaises, Champion, « B.G.F. », vol. I7, p. I095.

44. Ah quel conte!, éd. citée, p. 807. Il s'agit du livre second, $\mathrm{II}^{\mathrm{e}}$ journée du manuscrit de Madrid publié en annexe.

45. Ibid., p. 808 .

46. Idem.

47. Recueil de ces Messieurs, Amsterdam, Chez les Frères Westein, I745, p. 373-374. 
d'utile; cela me réconcilie avec elle ". À la lumière de cette remarque, on peut apprécier le reproche adressé par le roi Autruche à Taciturne, évoquant l'hypothèse d'un tête-à-tête plus abouti avec la grue : «Enfin, vous ne me ferez pas croire qu'une femme qui vous adore ait été la première à vous parler géométrie ${ }^{48}$ ?"

Si l'on admet avec R. Jomand-Baudry que $\mathrm{M}^{\mathrm{me}} \mathrm{du}$ Châtelet se cache sous la figure de la grue, il faut donc que Taciturne, qui rechigne à admettre l'attraction, incarne l'un des opposants à la théorie de Newton, défendue par la marquise. Mais dans une autre version du conte, imprimée et datant de 1754 , le personnage de Taciturne est présenté comme un bel esprit qui, pour avoir clamé dans le monde ses qualités de Géomètre et de Physicien, passe pour être, aux yeux de toutes les femmes, et quoique détesté, «le premier homme de son siècle ${ }^{49}$ ". R. Jomand-Baudry propose Maupertuis comme clé la plus évidente de ce "personnage à géométrie variable ${ }^{50}$ ", à côté d'autres propositions (La Mettrie, Maurepas, le duc de Richelieu), mais cette interprétation ne vaut pas pour la version manuscrite du conte. Taciturne a donc changé de camp entre 1739 et 1754 (date de la dernière version du conte). Si l'on se laisse prendre au jeu de devinettes qu'est le déchiffrage des clés, ne peut-on y voir une caricature de Cassini III ? Après avoir défendu, dans la lignée de ses ancêtres, des positions antinewtoniennes contre Maupertuis, il avait reconnu publiquement en 1744 ses erreurs. Cela constituait un jalon important dans la victoire des théories newtoniennes. La question de la forme de la Terre, qui était au cour des préoccupations de Cassini, faisait l'objet d'une des plus vives contro-

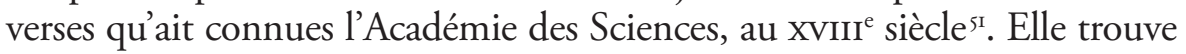
d'ailleurs sa répercussion burlesque dans le manuscrit de Ah quel conte!. C'est le même Taciturne qui parle :

J'ay des idées sur la figure de la terre, et je ne suis pas le seul. Beaucoup de gens parmy nous, la veulent absolument ronde; d'autres l'allongent, et veulent qu'elle ait à peu près la forme d'un melon aplati par ses deux bouts : quant à moy, je la crois octogone. Vous vous trompez tous, répondit gravement l'autruche ; je l'ay crû longtems octogone comme vous, mais le vray, est qu'elle est en forme de cône, autrement dit, en chapeau pointu ${ }^{52}$.

48. Ah quel conte!, man. de Madrid, éd. citée, p. 808.

49. Version de 1754, ibid., p. 302.

5o. Ibid., p. 699, note I2.

5I. Les mesures prises par les savants se contredisant partiellement, la querelle rebondit jusqu'en I756, puis le problème passe de mode (voir l'article «Terre » rédigé par D. Fauque dans le Dictionnaire Européen des Lumières, éd. M. Delon, Paris, PUF, 1997).

52. Ibid., p. 822-823. 
Il s'agit donc bien, pour Crébillon, d'inscrire son histoire dans l'air du temps, en parodiant les débats académiques et, dans les deux dernières versions d'Ah quel conte!, de se moquer aussi du roi Stanislas ${ }^{\text {er }}$ de Pologne, beau-père de Louis XV, qui s'était réfugié en France après avoir été dépossédé de son royaume, et été objet de risée en Europe. Passionné de sciences, il avait eu pour projet, en 1737 , de former une académie à Nancy et en avait fait la demande au roi. Cela avait suscité une autre controverse. En I750, alors qu'il est duc de Lorraine, il crée La Bibliothèque royale et la Société royale des Sciences et Belles-Lettres, future Académie de Nancy. Ces faits d'actualité justifient le fait que Crébillon, dans ses deux dernières versions du conte, modifie son personnage du roi Autruche qui, dans celle de Madrid, n'était qu'un bel esprit. Ce roi se livre, dans la version de 1754, à de plaisantes expériences astronomiques qui tournent au burlesque : ici c'est un cerf-volant qu'on envoie en l'air, avec des chats attachés, qu'une fée cachée dans un nuage foudroie de colère ${ }^{33}$. Là ce sont encore des cerfs-volants attachés aux étoiles par un génie, qu'il bat les uns contre les autres, provoquant un feu d'artifice, ce qui sert d'explication à l'origine des comètes. Le roi Autruche valide cette théorie, arguant ceci :

Il n'y a pas un Astronome de ceux qui ont écrit sur les comètes, qui n'en parle fort différemment de moi : et ce qui prouve combien il faut se défier des observations c'est qu'il y en a quelques-uns dont les idées sont assez raisonnables pour que l'on puisse les admettre ; quoi qu'assurément il n'y en ait pas un qui, sur cet article, ne se soit bien écarté du vrai ${ }^{44}$.

Les comètes avaient été en effet l'objet de thèses contradictoires chez les scientifiques, mais c'est évidemment ici l'explication délirante du phénomène qui est donnée pour vraie. Quoi que ce ne soit pas ici le sujet, ce propos abrite peut-être la justification des contes les plus parodiques, ceux qui cultivent, par exemple, le paradoxe de héros stupides faisant l'éloge du conte : la valeur culte qu'est la raison est un leurre, qu'il est possible de retourner contre ceux qui la défendent, les hommes de science, en prenant pour preuve leurs échecs et leurs querelles.

Aussi, dans ce conte - et bien au-delà - « la croisade de la science pour faire reculer la merveille est vaine. C'est elle qui aura le dessus, même si la dérision est toujours présente ${ }^{5 s}$ ", conclut Régine Jomand-Baudry. Il est vrai que l'auteur a choisi pour épigraphe de son conte politique et

53. Ces expériences sont rapportées dans la $4^{\mathrm{e}}$ partie, ch. 8 et 9 de la version de 1754 .

54. Ibid., p. 44I.

55. Ibid., p. 285 . 
astronomique un extrait des Satires de Perse : " $\hat{O}$ quantum est in rebus insane $^{56}$ " (Ô combien la réalité est vide).

\section{La focalisation critique ou l'école du regard}

Dans la fiction préfacielle des Aventures d'Abdalla de Bignon, la Lettre de Monsieur Sandisson au traducteur, un pseudo-éditeur, qui se présente comme un grand amateur de contes français, sollicite la créance du futur traducteur à qui il envoie ces récits originaux, si difficiles à croire. Il dit avoir été finalement convaincu de leur authenticité lors d'une aventure prodigieuse, similaire à celle vécue par l'un des personnages des récits, qu'il raconte : enlevé dans les airs, emporté dans un tribunal de péris et périses (bonnes fées orientales, à l'inverse des divs, mauvaises), il a assisté à la condamnation des premiers auteurs féminins de contes de fées français, puis à un second procès, celui de Gabalis, héros de Montfaucon de Villars. On sait que son roman Le Comte de Gabalis ou Entretiens sur les sciences secrètes ( 1670 ) a considérablement marqué la littérature et précisément, le conte de fées. Le criminel avoue son imposture et fait confession de ses mensonges, avant d'être condamné à mort et de disparaître dans la gueule d'un monstre. Cette scène de l'exécution capitale a été représentée, de plus, par un illustrateur anonyme, pour en dramatiser l'impact, et la gravure placée à un point stratégique de l'édition originale, en frontispice ${ }^{57}$.

On ne s'étonnera pas que la superstition, le mensonge des fausses sciences, l'occultisme, soient dénoncés par un religieux, mais on peut sourire devant le fait qu'ils soient punis par un prodige, d'autant que ce récit a pour fonction alléguée de servir de caution aux récits suivants, annoncés comme vrais quoique peu crédibles. L'adhésion demandée est évidemment sapée par le caractère invraisemblable et fantaisiste de cette mise en scène allégorique, ce qui ne veut pas dire que toute leçon, tout message qu'elle diffuse bénéficient du même coefficient de vérité ou d'erreur. L'indice de réfraction est différent, par exemple, pour ce qui concerne la critique du merveilleux pratiqué par les premières conteuses, et celle qui fustige l'usage d'un merveilleux hérétique (plus insupportable à un prêtre

56. Ibid., p. 299.

57. Elle est reproduite en vis-à-vis de la p. 952 de l'éd. citée (supra, note 8). J'analyse également, plus rapidement, cette illustration, en soulevant d'autres paradoxes propres au dispositif péritextuel de l'œuvre de Bignon, dans mon compte rendu du vol. 8 de la B.G.F. publié dans Féeries, n ${ }^{\circ} 4$, p. 254-255. 
que la gratuité du premier, ici jugé puéril, futile), ou encore enfin ce merveilleux oriental qu'il utilise lui-même.

Ce paradoxe de surface invite le lecteur à en chercher le sens. En lisant l'œuvre, en découvrant sa dimension apologétique, on comprend que le merveilleux qui a droit de cité chez Bignon, comme R. Robert le révèle dans l'introduction de son édition, c'est celui qui est asservi à cette intention, à la quête de la vérité. Car le lecteur doit prendre garde au point de vue qui oriente ce dispositif préfaciel, aux filtres utilisés. Le message qui lui est délivré est que toutes les fables ne sont pas bonnes à croire, même s'il l'est, paradoxalement, au travers du prisme déformant d'une fable. Si le lecteur n'est pas ce «traducteur » à qui M. Sandisson (double fictif de l'auteur) s'adresse, il se pourrait bien qu'il soit invité à en être un autre. Il doit se situer à un autre endroit que celui indiqué par le pseudo auteur à son destinataire fictif, surplomber l'ensemble pour mieux voir, et questionner cette vérité qu'on demande à l'éditeur fictif - et non à lui, lecteur - de croire. C'est l'incohérence du message, la divergence de ses éléments, l'aberration, qui le met sur le chemin. Lumineuse aberration, d'où naît le sens, voilé. Le dispositif énonciatif, tout comme le caractère métafictionnel du conte, suscitent le doute. Le lecteur va-t-il se laisser prendre au piège de cette représentation visuelle offerte par la gravure choisie pour frontispice au recueil de Bignon dans l'édition originale, qui a pour but de capturer son regard, tout comme le monstre sa proie, et de profiler sa lecture de l'œuvre ? Tout est fait, ici, pour cela.

Située au premier plan, à gauche, l'énorme bête (dont on ne voit que la tête - elle occupe le quart de l'image) fascine par sa taille, son globe oculaire exorbité, sa gueule ouverte prête à dévorer la victime ${ }^{58}$. Celle-ci est au même niveau qu'elle. L'homme paraît tout petit à côté, il est reclus dans le bas droit de l'image. Terrifié, il détourne la tête du monstre, il regarde à l'extérieur du cadre, comme s'il cherchait à s'échapper, coincé par l'angle de la gravure. À l'arrière plan, les spectateurs, assis en hauteur dans les gradins du tribunal, sont répartis de droite à gauche, en cercle. Les regards de la majorité d'entre eux convergent vers le centre de la scène offerte au premier plan. Mais, détail à considérer, ils n'ont pas tous la tête tournée vers le spectacle d'horreur - seraient-ils distraits ? Et ceux qui

58. Sa ressemblance avec les monstres infernaux dévorant les damnés, chez Hans Memling (L'Enfer, I485) et surtout Lucas Van Leyden (Le Jugement dernier, I526), est frappante. Le graveur semble avoir copié ce dernier tableau, reproduisant jusqu'au cadrage de la gueule monstrueuse. Si le référent religieux est refoulé du texte préfaciel de Bignon, la gravure le réintroduit allusivement. 
l'ont ne disposent évidemment pas du même angle de vue sur la scène qu'ils surplombent : l'image ne délivrerait-elle pas, par leur intermédiaire, le reflet en miroir d'une lecture distanciée, un mode d'emploi, d'autant que l'effet de perspective créée par le dessin met le lecteur, spectateur de la gravure exactement face à eux, et à la même hauteur, approximativement ? Exactement au centre de l'image, la reine des péris, placée debout dans l'arène, baguette magique à la main, semble fascinée par cette scène, qu'elle observe bouche-bée, bras écartés, tout en l'orchestrant de sa baguette (c'est elle qui a donné à Monsieur Sandisson cet ordre : "Vois, écoute, retiens et publies9 " qui justifie le rapport fait en préface, et donc l'œuvre qui la suit).

La scène se passe dans un amphithéâtre (lieu d'une expérience - de vivisection ?), qui est aussi un cirque (celui à la fois d'une mise à mort orchestrée et d'un jeu fantaisiste). Image globalement dominée par des lignes courbes, qu'elles soient lignes de force ou de détail. Le mur du fond, clôturant l'espace, est constitué d'une colonnade en arcades. Il est formé de deux ensembles de courbes, délimitant l'architecture de sa base, et celle de son sommet. Au centre de l'image, un autre ensemble de courbes dessine le balcon où sont amassés les spectateurs et qui sépare l'arène des gradins. Mais la perspective choisie par le dessinateur, pour donner au lecteur l'illusion de surplomber la scène, a nécessité l'inversion de ces courbes : les unes sont concaves (le balcon), les autres convexes (la colonnade du fond). L'image peut donc être assimilée à un dispositif optique, associant des lentilles des deux types. C'est donc bien une lunette, encore une fois, que l'auteur tend au lecteur à l'orée de son ouvrage. Ce frontispice est une image (une allégorie) stylisée, une projection circonscrite d'une scène "vue ", spectacle retransmis, mis en perspective et recomposé (même si l'anonymat de l'illustration a tenté de le faire oublier), une anamorphose. Elle fait réfléchir à ce qu'est la vision, aux déformations et aux illusions de nos sens. C'est d'autant plus intéressant, évidemment, qu'il s'agit de montrer précisément l'instant précédant la disparition d'un homme passé maître en illusions et mirages. Rapportons-nous au récit, en miroir : quant juste avant l'exécution, Gabalis passait aux aveux, s'adressant aux péris, il insistait sur la bonne vue des hommes qui ne se sont pas laissé duper par ses artifices :

J'avoue que j'ai fait tous mes efforts pour donner un nouveau lustre à un art que vous condamnez [...]. J'ai fait passer la magie noire, cette science que les divs enseignent,

59. Ouvr. cité, p. 947. 


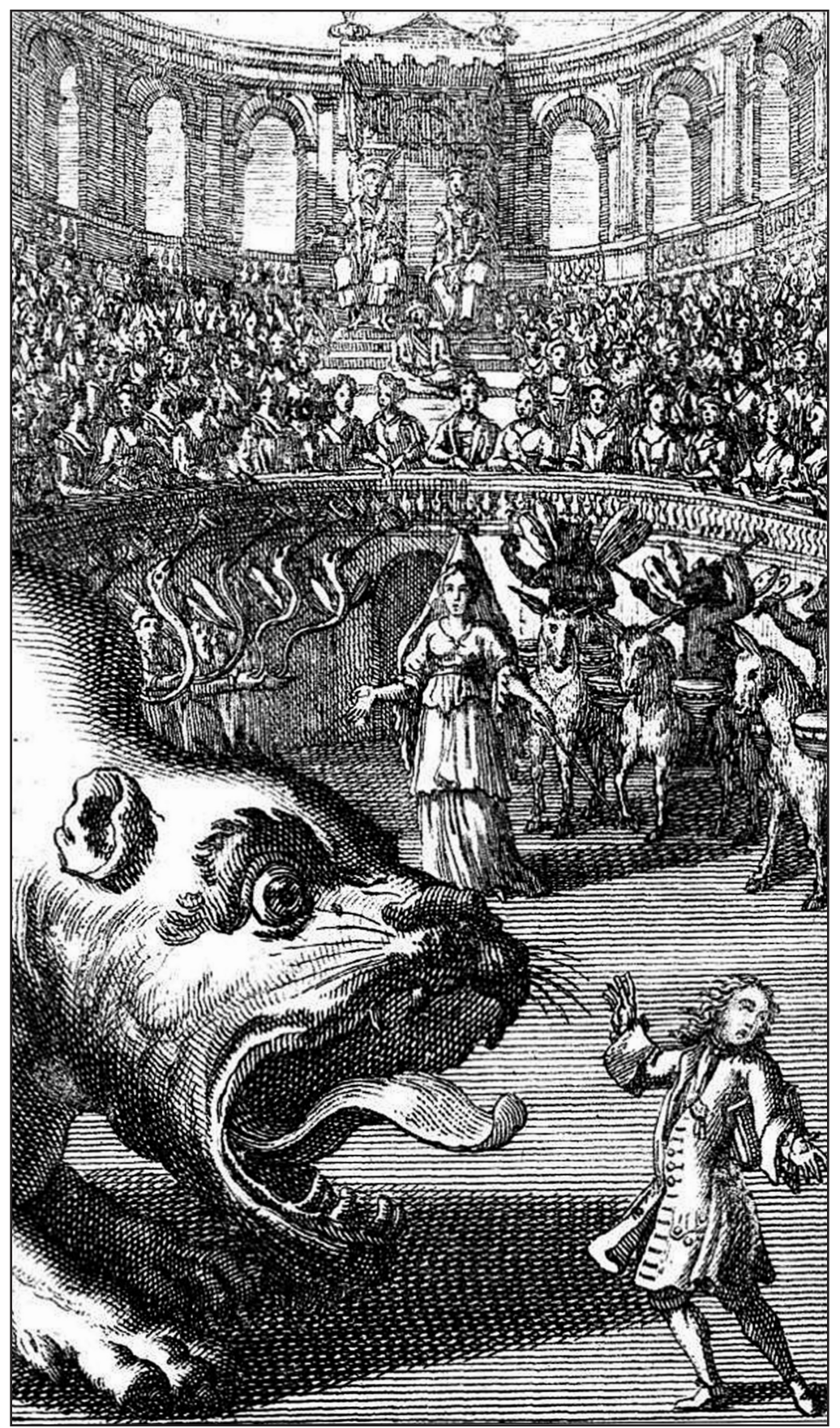

Fig. - Gravure placée en frontispice de l'édition originale des Aventures d'Abdalla de l'abbé Bignon. 
pour la véritable sagesse [...]. Au commencement, je travaillais à vous confondre avec les divs, mais sans succès, parce que les hommes ne purent ni vous discerner dans les maléfices des divs, ni reconnaitre les divs dans vos bienfaits ${ }^{60}$.

Après sa dévoration, le monstre sera lui-même aussitôt englouti dans le sol, raconte Sandisson : l'auteur aura réussi, en éliminant de cette façon le coupable, à faire passer les sciences occultes pour une éclipse de la pensée, définitivement révolue, son œuvre inaugurant une ère nouvelle, celle des Lumières.

Revenons au point de départ, à cette virulente attaque contre les sciences adossée à une réhabilitation du conte, que j'ai citée tout au début de cet article et qu'il est utile de replacer à présent dans son contexte. À qui doit-on ces propos qui ouvrent Le Sopha et figurent dans une "introduction " séparée du conte ? À un narrateur inconnu. Il vient de présenter rapidement son personnage, Schah-Baham, prince des Indes et petit-fils de Schah-Riar « de qui on a lu les grandes actions dans Les Mille et Une Nuits ${ }^{61}$ ", " bon et commode mari " quant lui, cocu sans le savoir, et bien peu savant de toute façon semble-t-il, puisque « le Recueil de Schéhérazade [...] était le seul livre qu'il eût jamais daigné lire ». C’est alors que ce portrait-charge s'interrompt pour laisser place à la réflexion citée, cet éloge du conte au déficit de la science. Le narrateur enchaîne alors sur la défense du sultan. Il y voit « un exemple bien mémorable de l'injustice des hommes à cet égard ${ }^{62} »$ :

Quoi qu'il sût l'origine de la féerie, aussi bien que s'il eût été de ces temps-là, que personne ne connût plus particulièrement le célèbre pays du Ginnistan, et ne fût plus instruit sur les fameuses dynasties des premiers Rois de Perse, et qu'il fût, sans contredit, l'homme de son siècle qui possédât le mieux l'Histoire de tous les événements qui ne sont jamais arrivés, on le faisait passer pour le Prince du monde le plus ignorant $^{63}$.

Aussi l'éloge du conte et son corolaire, la critique des sciences, sont-ils portés par un narrateur préférant d'entrée de jeu adhérer explicitement aux valeurs d'un personnage qu'il présente pourtant sous un jour peu amène, antihéros que le récit ne va cesser ensuite de ridiculiser. Cette situation narrative paradoxale est typique de cet esprit parodique qui caractérise notamment les conteurs, et se traduit par l'autodérision, la dévalorisation

6o. Ibid., p. 951.

6I. C. Crébillon, Le Sopha, conte moral, ouvr. cité, p. 28I.

62. Ibid., p. 282.

63. Idem. 
du genre qu'ils pratiquent. Ce qu'il faut noter ici, c'est la manière dont cette mise à distance ludique s'ingénie à taquiner le lecteur, brouillant sans cesse son regard, le ballottant entre divers points de vue : celui tout d'abord d'un premier narrateur, qui feint d'être aveugle sur la bêtise de son héros au moment même où il la met en lumière, en opposant ses idées au point de vue des détracteurs des contes. Le narrateur réhabilite alors les contes, dénonçant « l'idée de frivolité que l'orgueil et l'ignorance ont attachée à ce genre $^{64} »$. Puis, il cède sa place à un second, censé avoir écrit une Histoire des Indes, dont les aventures qui vont suivre sont tirées. Celui-ci adopte le point de vue inverse du précédent. Il rapporte en direct une conversation entre la sultane et son époux. À partir de ce moment, tout est "montré » au lecteur, que le discours direct place en position d'observateur objectif: la sultane lapide les contes et Schah-Baham tente, en bredouillant, une piteuse réfutation, ajoutant un nouveau couplet à la défense du conte formulée plus brillamment par le premier narrateur. Et le sultan de conclure :

Puisqu'un conte m'a toujours amusé, il est clair qu'il faut qu'un conte ne soit pas une chose si frivole. Ce ne sera certainement pas à moi qu'on fera croire qu'un sultan peut être une bête ${ }^{65}$.

Parvenu à ce stade, le lecteur est totalement convaincu de l'inverse. La répétition parodique de l'éloge du conte dans la bouche du sot a achevé de dégrader les théories développées par le premier narrateur - et par rebond, l'auteur qui s'adonne au genre.

Ces relais de discours performés par des voix tour à tour convergentes et divergentes, se renvoyant la balle à des niveaux structurels distincts de la narration, mettent à mal le lecteur qui chercherait le " vrai " point de vue, le plus juste, tant la thèse, s'il en est une, est fuyante. Elle suit un parcours complexe, on l'a vu, à travers une série de lentilles concaves/convexes (les personnages et narrateurs), ricochant sur des miroirs, ou ces dioptres que sont les décrochages narratifs et discursifs (emboîtements, dialogues, récits les surplombant). Les jugements de valeur - appelés justement réflexions y fusent, suivant un parcours en zigzag fait d'inversions successives, comparable à celui des rayons lumineux dans un instrument d'optique. Encore une fois, le lecteur est sommé de chercher la bonne distance, invité à pratiquer lui-même d'incessantes mises au point. Ces brouillages lui imposent littéralement d'accommoder sans cesse. Cette gymnastique oculaire est un exercice utile pour bousculer les préjugés, affuter l'esprit critique.

64. Idem.

65. Ibid., p. 287. 
La régression calculée vers le puéril et l'irrationnel opérée par ces auteurs de contes de fées qui jouent les sots a besoin d'une valeur ajoutée pour compenser le manque à gagner qu'on leur a parfois reproché du côté du sérieux, voire de l'intelligence. Le conteur fait la démonstration de ses pouvoirs critiques en moralisant, en ironisant, éblouissant le lecteur, parfois, de ses jeux d'esprit malicieux. Pour échapper à la ridicule prétention qu'il fustige souvent, celle des savants ou des beaux esprits, il procède en biaisant : il se moque de lui-même. Le degré de vérité des énoncés étant ébranlé par la fiction, la futilité du genre, l'ironie des narrateurs, le conte a besoin de ces ingénieux dispositifs énonciatifs pour susciter le doute et l'entretenir chez le spectateur. Le discours féerique ne renonce pas au mensonge pour dénoncer le mensonge, il sait que de cette posture métacritique peut naitre une vérité, qui ne prétend pas être la vérité pleine et entière, mais vérité en construction, vérité relative. Ainsi, le conte, cette machine optique, ce microscope, place-t-il le lecteur dans la position du savant qui expérimente en faisant d'abord table rase de ses certitudes.

Aussi, quand les auteurs réfléchissent, en les déformant, les débats scientifiques et les inventions, raillent ces expériences ridicules qui se soldent par un échec, quand ils se moquent du vide des sciences, ils témoignent plus que jamais de cet élan vers les Lumières, et rappellent que le savoir n'est jamais chose figée, définitive. Fontenelle, évoquant la manière fabuleuse dont les anciens Chinois expliquaient le phénomène des marées, ironisait : "Vous jugez bien qu'ils n'iront pas penser à la pression de la lune sur notre tourbillon ${ }^{66}$. " Or au moment où est publié ce texte, les théories cartésiennes sont déjà assimilées par quelques jeunes savants à de vulgaires fables. La frontière est décidément poreuse, entre science et fable (ou conte).

66. Fontenelle, De l'origine des fables, éd. citée. 